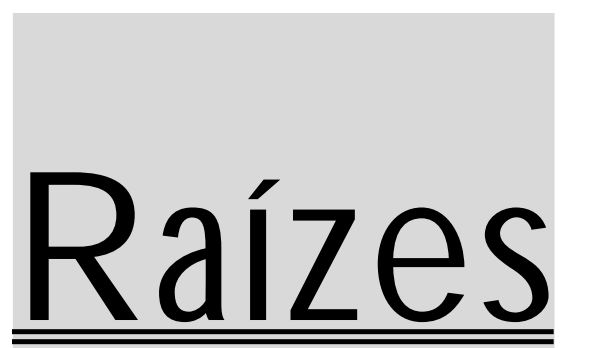

v. 28, ns. 1 e 2, jan. - dez./2009; v. 29, n. 1, jan. - jun./2010

D ossiê: Território, sustentabilidade e ação pública

(M arc Piraux, Márcio Caniello - O rgs.)

\title{
ARTICULAR DISPOSITIVOS DE FORMAÇÃO E DE GOVERNANÇA: UM DESAFIO PARA O DESEN VOLVIMENTO ${ }^{1}$
}

\author{
$\overline{\text { E milie C oudel, Jean-Philippe Tonneau, M arc Piraux }}$
}

\begin{abstract}
RESU MO
0 desenvolvimento territorial enfrenta muitas vezes a falta de competência para favorecer o seu desempenho. As formações são meios freqüentem enteu sados para melhorálas. E sta comunicação propõe uma reflexão sobre a maneira como formações podem contribuir ao fortal ecimento das capaci dades necessárias no âmbito de um desen volvimento territorial. Reforçar, simul taneamente, o capital humano, social einstitucional dosatores constitui um verdadeiro desafio para uma formação. A poiando-nos sobre teorias da aprendizagem, sugerimos hipóteses demelhoramento dos dispositivos de formação: a diversidadedos atores envolvidos, a necessidade de uma probl ematização e a inserção da formação nos processosterritoriais. D estacamos, especialmente, a importância de vincul ar os dispositivos de formação aos dispositivos de governança territorial para permitir aos atores exercer plenamente as novas competências adquiridas.
\end{abstract}

Palavras- chave: competências, formação, UniversidadeC amponesa

\section{TO LINK GOVERN ANCE AND COMPETEN CIES DEVICES: ONE CH ALLENGE FOR THE TERRITORIAL DEVELOPMENT}

\section{ABST RACT}

Territorial development faces lack capacities. Formation is often used to improvethesecapacities. This communication proposes a reflexion how to reinforce the capacities necessary to territorial development. These capacities are diverse, and reinforcing simultaneously the human capital, social capital and institutional capital of theactors constitutes a real chall enge for a training process. U sing differentlearning theories, wewill suggest hypotheses concerning training for sustainable devel opment: the diversity of actors, problematic and theinsertion of the process in theterritory. We will stress in particular the importance of linking the training devices to the devices of territorial governance, to enable the actors to fully apply the new competencies acquired to the benefit of territorial devel opment.

Key words: competencies, capacity building, PeasantU niversity

E milieC oudel. D outor em economia, U M R Innovation, M ontpellier SupAgro, France, emilie.cou del@ cirad.fr. Jean-PhilippeTonneau.D ou tor em geografia.CIRAD, M ontpellier France, jean-philippe.tonneau@ cirad.fr. Marc Piraux. D outor em agro-economia, CIRAD, professor visitante na UFCG, marcpiraux@ uol.com.br.

1 Texto apresentado no Seminário Internacional "D esenvolvimento Sustentável eTerritórios Rurais: Quais desafios para a ação pública", organizado pel o Cirad, a U niversidade Federal de Campina Grandee a Fundação U niversidade Camponesa realizado em Campina Grande/PB no período de 22 a 25/09/2009. 
INTRODUÇÃO

Enquanto a descentralização se impõe gradualmente desde a década de 1990 no mundo inteiro, levando a uma transformação das lógicas de desenvolvimento e de governo, o território aparece como uma nova área de governança, entre escalas locais e estaduais, entre dinâmicas locais e diretivas legislativas, permitindo mais ligações entre o governo e a sociedade civil, articulação necessária para desenvolver um projeto de território comum (D effontaines et al., 2001). N ovas políticas públicas e novos dispositivos de planejamento territorial ( $\mathrm{L}$ ardon et al., 2008) são implementados para facilitar esta governança. M as a capacidade real, no sentido das capabilidades (capability) de Sen (1989), dos atores a participar nestes processos constitua ainda um problema. 0 governo brasileiro, em decidir criar territórios pilotos para o desenvolvimento da agricultura familiar, se depara com este desafio (SDT /M DA, 2005a). Ele quer fortalecer a participação popular, mas, na prática, esta participação é muitas vezes limitada, em parte devido à falta de competência dos agentes locais.

Em 2003, uma experiência da U niversidade Camponesa foi proposta pelo $\mathrm{CIRAD}^{2}$, a UFCG ${ }^{3}$ e o projeto D om H elder Camara ${ }^{4}$ para fortalecer a capacidade dos líderes rurais para propor seus próprios projetos e participar das políticas territoriais. Esta formação aconteceu no Cariri Paraibano e foi estruturada como um espaço de construção da identidade, de competências, de capital social e de afirmação de um projeto comum (Caniello et Tonneau, 2006). A formação foi replicada num outro contexto (no Território do Alto Sertão Piauí/Pernambuco-TASPP numa parceria C irad-E mbrapa ${ }^{5}$ ). L ogo, essas experiências tiveram sucesso, os estudantes criaram uma associação para garantir a continuidade da experiência e agir sobre 0 desenvolvimento da região. Porque será que estas experiências tiveram tantos impactos sobre os atores? 0 que aconteceu? 0 que aprenderam os atores? Para responder a estas perguntas, um doutorado foi iniciado para abordar a questão mais ampla das aprendizagens para o desenvolvimento terri- torial (C oudel, 2009). 0 objetivo era esclarecer quais são as aprendizagens necessárias para permitir a inserção dos atores nas dinâmicas de desenvolvimento territorial.

N este artigo, propomos apresentar o quadro teórico desenvolvido durante o doutorado para analisar as contribuições das formações e confrontá-las à visão do desenvolvimento territorial proposto pela Secretaria do desenvolvimento territorial (SDT) do Ministério do D esenvolvimento Agrário (M DA) (SDT/M DA, 2005a). $\mathrm{N}$ a primeira parte, identificamos os desafios da capacitação dos atores, especialmente em respeito à gestão social dos territórios (SD T/M DA, 2005b). N a segunda parte, apresentamos diferentes abordagens que permitem a formalização das aprendizagens, para formular hipóteses sobre a maneira de acompanhar aprendizagens para o desenvolvimento territorial. Finalmente, na terceira parte, propomos refletir sobre os el ementos que determinam o sucesso de ações visando a reforçar as competências para o desenvolvimento territorial. Concluímos, salientando a importância de ligar os dispositivos de formação aos dispositivos de governança territorial para permitir aos atores exercer plenamente as novas competências.

\section{REFORCAR AS CAPACIDADES DOS ATORES}

\subsection{O DESAFIO DA GESTÃO SOCIAL}

A referência conceitual que norteia a dimensão político-institucional do desenvolvimento dos territórios rurais no B rasil (SDT/M DA, 2005b) é a G estão Social. É entendida nos documentos oficiais da SDT como "um processo de gerir assuntos públicos, por meio da descentralização político-administrativa, redefinindo formas de organização e de relações sociais com sustentabilidade, trans parência e efetiva participação da sociedade, o que implica ampliação dos níveis das capacidades humanas, sociais e organizacionais do Território" (SD T/M DA 2005b).

0 principal objetivo é estabelecer mecanismos que possam trazer a sociedade civil dentro da esfera política, para interagir com os poderes públicos. Busca-se uma

\footnotetext{
2 CIRAD : Centro Internacional de Pesquisa em Agronomia para o D esenvol vimento

3 UFCG: Universidade Federal deCampina Grande.

4 Projeto D om H el der Camara: Fundação juntando o Ministério do D esenvolvimento Agrário e o FIDA (Fundo Internacional de D esenvolvimento da Agricultura) que desenvolve políticas experimentais de credito para o semi-arido brasileiro.

5 EMBRAPA: Empresa Brasileira de Pesquisa Agro-Pecuaria.
} 
ruptura nas práticas de gestão setorial, centralizadoras e pouco participativas que, de forma geral, são ainda comuns na administração pública. Espera-se que por meio dos princípios de democracia e participação «a gestão social pode ser não apenas um instrumento de controle público sobreas políticas os recursos que elas permitem investir, mas também um instrumento que leva ao empoderamento da sociedade» (M DA/SDT 2005b, p: 13). Assim, essa integração da sociedade civil tem dois objetivos: primeiro, permitir novas relações entre as esferas políticas, sociais e econômicas; segundo, levar ao seu empoderamento para exigir compromissos com os poderes públicos.

Assim, com a noção de gestão social , a política do SDT visa a fomentar a constituição de espaços de governança, a partir da criação de novas instituições e a negociação de regras comuns para a implementação de projetos. D e fato, a SDT propõe traduzir o processo numa realidade concreta mediante a elaboração de projetos, guiados pelas grandes linhas definidas num Plano de D esenvolvimento Territorial Rural Sustentável (PDTRS).

E spera-se que, ao final desse ciclo, sejam alcançados diferentes produtos: o diagnóstico, o plano territorial, um processo de planejamento, projetos de dimensão e de natureza territorial, objetivos e metas monitorados. 0 ciclo visa também mudanças sociais como o reconhecimento e a mobilização do capital social, arranjos institucionais e um «sistema de gestão social retro-alimentado», com agentes locais que dominam o processo (Figura 1).

\section{CICLO DA GESTÃO SOCIAL}
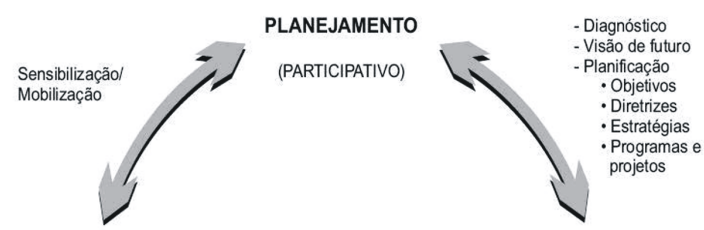

CONTROLE SOCIAL

(TRANSPARENTE)

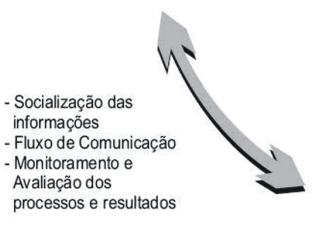

ORGANIZAÇÃO (DESCENTRALIZADA)
Figura 1. Ciclo da gestão social proposto pela SDT.
O SDT afirma nos documentos orientadores (MDA/ SDT, 2005b) que é preciso "catalisar habilidades e capacidades" e dar "capacitação às organizações do território". $\mathrm{N}$ a visão de gestão social, o desenvolvimento territorial é visto como um processo que contribui a desenvolver as capacidades a elaborar, implementar e monitorar os projetos escolhidos. Implicitamente, é esse desenvolvimento de capacidade que deve contribuir ao empoderamento das populações definidas como o meio pelo qual as pessoas adquirem maior controle sobre as decisões que afetam suas vidas (L averack e L abonte, 2000). Assim, é pressuposto que "com capacidades", os atores devem se empoderar do processo e levar ele para frente com projetos... M as quais tipos de capacidades?

\subsection{O DESAFIO DAS COMPETÊNCIAS NECESSÁRIAS AO DESEN VOLVIMENTO TERRITORIAL}

Q uais são as competências necessárias para o desenvolvimento territorial, ou mais precisamente, quais são as capacidades que um ator precisa para se envolver no desenvolvimento territorial? A partir do estado da arte realizado durante o doutorado sobre esse assunto, confrontado com as entrevistas com atores envolvidos (técnicos, políticos, representantes de associações...), retermos diferentes tipos de capacidades.

Para permitir a um processo comum de surgir, como formalizado no ciclo da gestão social, é necessário ter uma capacidade de interação para compartilhar conhecimentos com os outros (ouvir e falar), para aprender com os outros (abertura para novos conhecimentos), para envolver novas pessoas nas discussões, para resolver conflitos. Em seguida, para propor projetos adequados, deve-se ter uma capacidade de análise, ou seja, saber mobilizar conhecimentos existentes, construir novos conhecimentos, ter um olhar crítico sobre esse conhecimento. Esta capacidade de análise deve levar ao desenvolvimento de uma capacidade de concepção e programação de projetos inovadores, mas também realizáveis. Para implementar e gerenciar os projetos é preciso ter uma capacidade de realização e de gestão, as habilidades práticas para implementar e gerenciar projetos, mobilizar outros atores dentro das redes territoriais, para construir instituições. Finalmente, para completar o ciclo, é necessário ter uma capacidade de auto-avaliação e crítica, para fazer evoluir o projeto territorial.

Assim, as capacidades necessárias são diversas e exigem conhecimentos e habilidades (saber-fazer), também 
diversos: conhecimento acadêmico e saber-fazer prático, conhecimento técnico, econômico einstitucional que se traduzem em capacidades de ação ou de reflexão, individual, mas também coletiva. N o entanto, não basta ter a capacidade, também é necessário saber como mobilizá-la na ação. I sso é o que chamamos de "competência". A competência é o poder de agir, não em termos absolutos, mas de acordo com uma determinada situação (Z arifian, 2001). As competências, "saberes-fazeres" de al to nível, permitem enfrentar uma situação singular e complexa, pela concepção, organização, estruturação, avaliação, restituição, para elaborar respostas adequadas e não simplesmente reproduzir respostas estereotipadas (Perrenoud, 2001).

Este desenvolvimento de competências pode ser gradual, mas é raramente espontâneo, daí a importância de estabelecer um processo de acompanhamento (Lardon et al., 2007).

\subsubsection{DESAFIO DO ACOMPANHAMENTO DAS APRENDI-} ZAGENS

O uso da palavra "acompanhamento" é bastante recente nas disciplinas ligadas ao desenvolvimento rural. Ele foi inicialmente usado em ciências sociais, para falar de apoio social às pessoas em situação de dificuldade, ou em ciência da gestão, para descrever um processo de "coaching" de empresas.

$\mathrm{N}$ a área de desenvolvimento rural, a expressão "acompanhamento" caracteriza um processo em que o ator está no centro do processo. Esta expressão vai além de uma visão de "apoio" ou de "consel ho", termos habitual mente utilizados conjuntamente com as idéias de "difusão" ou de "transferência" de conhecimento. 0 acompanhamento liga um ou mais "acompanhados" com um ou mais "acompanhadores", e o acompanhado que faz a solicitação. Ele é voluntário num processo cuja responsabilidade é dele (Amoureux, 2002). 0 objetivo do acompanhamento é que 0 ator adotasse uma postura reflexiva que permite 0 desenvolvimento da aprendizagem. Esta concepção do acompanhamento é similar à abordagem que K nowles (1990) propunha para a educação de adultos. Para Knowles, um adulto tem uma necessidade psicológica de ser reconhecido como um ser que se determina a si mesmo (self-directing). É necessário levar em conta a experiência e negociar um projeto de formação baseada em elementos de interesse na vida real - Se não for o caso, o acompanhado não vai aderir ao projeto de formação. Antes de tudo, o papel do acompanhador é criar as condições que permitem superar as dificuldades do processo, devido o fato de que as aprendizagens devem ser tácitas e coletivas.

A construção dos conhecimentos tácitos necessita num primeiro tempo ser livre de restrições (capital, mercado), mas deve depois levar em conta esta realidade.

A segunda dificuldade vem da dimensão coletiva. Segundo Argyris e Schön (1978), esta dimensão coletiva requer a construção de visões, valores, responsabilidades compartilhadas. D entro das empresas, e cada vez mais na área de enfoques territoriais (Callon, 1989), processos de pesquisa-ação são recomendados ( $\mathrm{H}$ atchuel, 2000). Permite tanto a aplicação na realidade como interações que podem dar origem às dinâmicas coletivas. Segundo Albaladejo e Casabianca (1997), mais do que uma co-produção de conhecimento entre dois mundos (por exemplo, entre a universidade e o mundo profissional), a pesquisa-ação permite a construção de mecanismos coletivos que podem gerir uma inovação contínua.

0 conceito de pesquisa-ação desqualifica as formas convencionais de formação. Estas se baseiam em conteúdo predeterminado, a serem apropriados individualmente pelos alunos em formação. Ao contrário, no acompanhamento, o importante é se adaptar à real idade dos acompanhados e estimular dinâmicas coletivas dentro do grupo. A generalização do processo de acompanhamento a outros contextos é um desafio real, porque depende da relação entre o processo e a sua inte gração territorial.

Para garantir esta capacidade de adaptação, diferentes teorias podem trazer elementos de reflexão para entender 0 acompanhamento das aprendizagens. Propomo-nos a adaptar à realidade do desenvolvimento territorial algumas teorias da economia do desenvolvimento e das ciências de gestão.

\section{COMO ANALISAR AS APREN DIZAGENS?}

\subsection{A ECONOMIA DO DESENVOLVIMENTO: COMO ENTEN- DER A DIVERSIDADE DAS APRENDIZAGENS?}

Para definir a natureza das competências adquiridas, tentamos descrever o caráter individual e coletivo das competências mobilizadas no desenvolvimento territorial. 
$\mathrm{N}$ as correntes da economia do desenvolvimento e da economia da educação, é comum usar o termo "capital humano" para caracterizar as competências no nível individual. A "teoria do capital humano" (Becker, 1993; Schultz, 1961) postula que o conhecimento acumulado pelos indivíduos, tais como o capital financeiro, permite criar riquezas.

Para superar uma abordagem individualista e abordar as competências coletivas, outros conceitos semeIhantes podem ser usados. Assim, o conceito de capital social surgiu seguindo o de capital humano, para caracterizar a contribuição das relações sociais (L in, 2005). $\mathrm{N}$ um primeiro tempo, ele foi definido a um nível individual, para explicar como os indivíduos podem investir e beneficiar-se das suas relações sociais ( $G$ ranovetter, 1985), mas ele foi progressivamente ampliado para a análise das dinâmicas sociais de comunidades e de nações, especialmente com as análises de Putnam (1993). $\mathrm{N}$ osso objetivo, sendo caracterizar as aprendizagens para o desenvolvimento regional, um terceiro nível de análise, parece necessário. É preciso ir além da análise do grupo e das redes para compreender o nível das organizações e instituições. Referimo-nos ao conceito de "capital institucional", embora ainda pouco utilizado. Krishna (1997) o define como um conjunto constituído por "elementos estruturais - papéis, regras, procedimentos e organizações - que facilitam a ação coletiva num benefício mútuo".

E mbora o conceito de capital geralmente tenha um conotação "econômica" e pode ser considerado limitante para ciências da administração ou para a sociologia, ele apresenta um interesse na sua capacidade em modelar um processo de construção e de mobilização. L in (2005), baseado na análise de $M$ arx (B rewer, 1984), distingue dois componentes: o capital é, ao mesmo tempo, o produto de um processo de produção (ele se acumula) e um recurso para investimento (ele é mobilizado). Estas duas vertentes valem para todos os conceitos de capital que têm surgido na linhagem do capital produtivo.

E stes conceitos podem, portanto, traduzir uma idéia de processo contínuo de construção e mobilização. A acumulação de capital (humano, social ou institucional) depende da qualidade do processo de acompanhamento. A mobilização destes capitais depende da relação com o contexto. D efato, seguindo a visão deSen (1989), não é suficiente deter um capital para poder usá-lo, é também necessário que 0 ator tenha a oportunidade de fazê-lo.
Os conceitos de capital humano, capital social e capital institucional permitem ligar as competências e motivações individuais, as relações sociais, aos valores e conhecimentos coletivos, assim como à governança territorial e os seus arranjos institucionais. Para entender estes capitais em termos de processos de aprendizagens, é preciso recorrer às análises desenvolvidas pelas ciências de gestão.

\subsection{AS CIÊNCIAS DE GESTÃO: QUAIS PROCESSOS DE APRENDIZAGEM?}

$\mathrm{N}$ as ciências de gestão, as teorias de action-learning analisam como as aprendizagens realizadas durante a ação podem levar a desenvol ver novas práticas. A póiamse nas teorias da psicologia comportamental (behaviorism), que postula que a aprendizagem acontece gradualmente, corrigindo os erros em função do resultado observado (ver Figura 2).

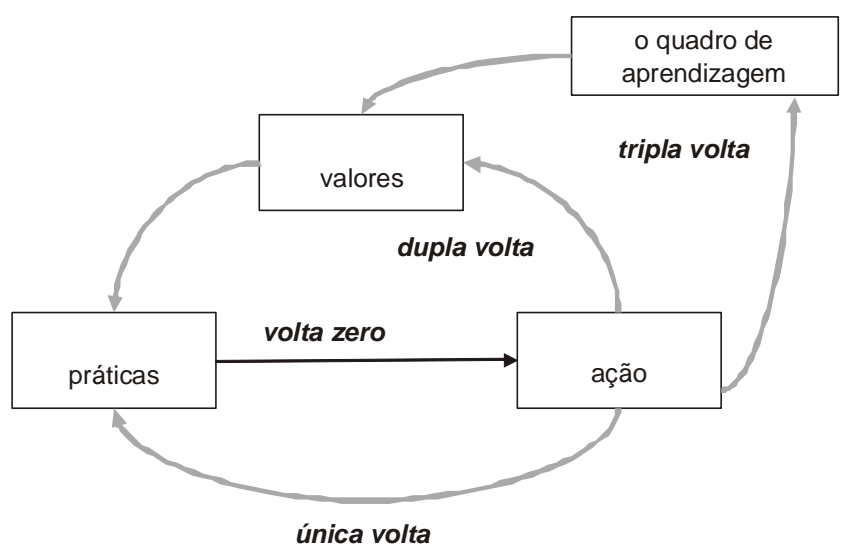

Figura 2. Voltas de aprendizagens.

N esta formalização, a aprendizagem é causada por um "estímulo", um fator de mudança. N uma situação relativamente estável, os atores integram gradualmente novas práticas, por simples transferência (volta zero). Se os atores notam uma perda de eficiência, eles podem tentar melhorar numa volta única de aprendizagem, onde eles desenvolvem coletivamente uma nova prática. Se esta aprendizagem não for suficiente para melhorar a situação, os atores são levados a ter uma aprendizagem em volta dupla, para desenvolver uma nova compreensão dos problemas e a uma nova maneira de resolvê-los.

$\mathrm{N}$ um contexto de mudança generalizada, os atores devem aprender a reagir e se adaptar às mudanças e, 
assim, desenvolver novas formas de aprendizagem em conjunto, através de aprendizagens em tripla volta. Este conceito é similar ao de "aprendizagem social" (social learning) desenvolvido pela ciência política para caracterizar processos de aprendizagens nas políticas e na governança (Turcotte et al., 2007) que questionam as normas sociais (Foldy et C reed, 1999).

A possibilidade de desenvolver estas voltas de aprendizagens em resposta ao estímulo do contexto depende das relações que existem entre os atores. A "volta zero" pode ser al cançada simplesmente numa relação de transferência entre dois indivíduos. A volta única se desenvolve através do intercâmbio entre al gumas pessoas. Para a volta dupla, Argyris e Schon (1996) consideram que é necessário que uma organização estável exista. A volta tripla seria enfim desenvolvida dentro de uma organização em constante movimento, efêmera (Romme et van Wittel oostuijn, 1999).

Assim, dependendo do estímulo e da forma das relações entre pessoas, várias mudanças podem acontecer, nas práticas ou nos valores. Esta formalização das aprendizagens permite analisar as mudanças e os seus determinantes, com um destaque especial nas relações.

\subsection{HIPÓTESES PARA GUIAR O PROCESSO DE ACOMPA-} NHAMENTO

A partir destas teorias, é possível destacar al guns princípios que deveriam orientar os processos de acompanhamento. Eles podem também guiar nossa análise dos processos, são então hipóteses que utilizamos para orientar a nossa ação-reflexão.

A nossa primeira hipótese é que para contribuir para o desenvolvimento territorial, é necessário ter uma aprendizagem em nível individual, em nível de grupos sociais e em nível institucional. Um processo de acompanhamento deve se preocupar com o desenvolvimento da aprendizagem nesses três níveis, quer dizer reforçar simultaneamente o capital humano, o capital social e 0 capital institucional.

A nossa segunda hipótese é que a aquisição da aprendizagem depende, em cada nível (individual, grupo, institucional) de fatores específicos. 0 processo pedagógico precisa assegurar as condições e os dispositivos que vão permitir favorecer cada tipo de aprendizagem. Diferentes voltas de aprendizagens podem acontecer, dependendo destas condições, levando a aprendizagem a cada nível. M as, estas condições não podem ser "prede- terminadas", elas devem ser adaptadas às aprendizagens necessárias. N este sentido, um processo de acompanhamento se define "caminhando", para permitir um processo adaptativo e inovador e construir progressivamente as condições necessárias.

U ma terceira hipótese pode ser acrescentada. Além de favorecer a aprendizagem e a construção dos capitais, é importante pensar na maneira como poderão ser utilizadas na ação (a mobilização dos capitais). D esde o início, deve-se inserir a aprendizagem na realidade para que a aprendizagem seja enraizada na realidade e que as condições da utilização futura das capacidades sejam definidas logo. A interação entre aprendizagem e realidade é o fundamento das voltas. Elas acontecem, na ação, permitindo a reflexão nas conseqüências da prática, e assim levam a desenvolver novas práticas para agir melhor.

A primeira hipótese se interessa principalmente pelos resultados da aprendizagem, enquanto a segunda hipótese está focada sobre o processo que levou a essa aprendizagem. A terceira hipótese leva a pensar essa aprendizagem numa perspectiva mais larga, inserida no território.

São estas hipóteses que permitem analisar o processo de aprendizagem, a partir do exemplo de duas U niversidades Camponesas.

\section{O ACOM PANHAMENTO DE APRENDIZAGENS}

3.1. 0 EXEMPLO DE DUAS UNIVERSIDADES CAMPONESAS

A U niversidade Camponesa no Brasil foi iniciada no final de 2002 por um grupo de pesquisadores do Brasil (principalmente do $\mathrm{N}$ ordeste), com a elaboração de uma Carta M agna, estabelecendo princípios guias. A intenção era permitir aos projetos independentes surgir em torno de idéias federativas: a participação das organizações de produtores no processo de formação ea co-construção de conhecimentos adequados à agricultura familiar e ao desenvolvimento sustentável. 0 objetivo das formações era formar jovens rurais para permitir-lhes conciliar inserção profissional, desenvolvimento comunitário sustentável e participação na construção das políticas públicas locais.

Em 2003, um projeto piloto chamado U nicampo foi proposto pela UFCG , o CIRAD e o Projeto D om H elder Câmara na região do Cariri da Paraíba (C aniello et al, 2003). Em 2004, outro treinamento foi lançado pela 
EMBRAPA e o CIRAD (Piraux et al, 2007) em uma região de fronteira entre o Piauí e Pernambuco. Estas duas formações foram desenvolvidas paralelamente à implantação de territórios. Com o decorrer do tempo, podem ser analisadas como ações de acompanhamento às dinâmicas territoriais.

$\mathrm{N}$ os dois casos, o processo pedagógico associou diferentes ciclos: o primeiro permitiu a sensibilização aos problemas de subdesenvolvimento na região semi-árida; o segundo foi centrado na formação pela pesquisa (análise das situações da região) e o último incentivou a elaboração de projetos individuais e col etivos, através de uma formação técnica e instrumental para responder às necessidades dos projetos. 0 dispositivo pedagógico da U nicampo era baseado na pesquisa-ação para inscrever o processo de aprendizagem na realidade, mas também para gerar dinâmicas nos grupos de pesquisa, constituídos de educandos e educadores, numa troca de igual a igual: os educandos trazem o conhecimento da realidade, os educadores trazem formalização e instrumentos de pesquisa (Caniello et Tonneau, 2006).

\subsection{QUAIS FORAM OS ELEMENTOS IMPORTANTES NAS} UNIVERSIDADES CAM PONESAS?

Seguidamente, analisamos quais foram os elementos importantes nestas formações que favoreceram as aprendizagens.

Diversidade, debate e construção de regras

Os processos pedagógicos foram fundamentais pela sua capacidade em estabelecer um clima de confiança e reciprocidade no respeito às opiniões expressas por cada um. A diversidade dos perfis dos atores, tanto para os educadores como para os educandos, foi um elemento decisivo, pois permitiu a descoberta e a troca de experiências entre pessoas.

As U niversidades $\mathrm{C}$ amponesas mobilizaram diversos perfis de formadores: antropólogo, psicólogo, sociólogo, geógrafo, engenheiro agrônomo. Essa diversidade foi mantida durante toda a formação. $\mathrm{N}$ ão era apenas para apresentar uma diversidade de pontos de vista, mas também para eles se enfrentarem. Os educadores estavam muitas vezes presentes às aulas dos colegas. As suas intervenções aceitas e encorajadas mostraram aos alunos, in vivo, que um educador não sabe tudo, que o saber pode ser debatido e que se constrói de forma interativa no debate.

O s estudantes, embora todos relacionados com a agri- cultura, apresentavam também uma grande variedade de perfis. D urante a formação, a variedade de pontos de vista permitiu ultrapassar os preconceitos, por exemplo, entre agricultores e assentados. D epois da formação, a diversidade das diferentes organizações onde os alunos se inseriram permitiu juntar várias redes, aumentando a possibilidade de circulação de informações dentro do território e contribuindo para a participação nos processos de governança.

A construção de regras permite ao grupo funcionar e desenvolver um sentimento de responsabilidade.

U m processo organizado em torno do questionamento

U $m$ dos principais pontos fortes da formação foi conseguir problematizar os princípios guia. D efinidos na Carta M agna da U niversidade C amponesa e adaptados em cada experiência, estes princípios valorizaram especialmente a identidade camponesa e promoveram a coconstrução do conhecimento.

M esmo na formação técnica, é necessário contextualizar, problematizar, não apenas a esfera técnica, mas a situação das pessoas na suas dimensões econômica, social e política. U $\mathrm{m}$ dos desafios é que os atores entendessem como funciona a sociedade local, para poderem perceber a necessidade e as modalidades para lançar uma ação coletiva.

No caso da Unicampo, os educadores operacionalizaram estes princípios com uma problematização durante o processo pedagógico, com sete perguntas-chaves: Q uem somos? 0 que temos? Como usamos o que temos? Como potencializar o uso do que temos? Qual é o nosso projeto? Como colocar o projeto em prática? Como gerir o projeto? Estas perguntas constituíram um quadro para estruturar toda a formação, orientando progressivamente as aulas. M esmo se tudo não estava determinado no início, a visão geral tinha sido escolhida.

O s conteúdos foram gradualmente elaborados a partir destas perguntas-chaves para construir o conhecimento durante a formação a partir da interação com os alunos. M esmo se o conteúdo tem a sua importância, e os formadores traziam sólidos conhecimentos científicos, o domínio dos princípios deve servir ao processo, não orientá-lo. D e fato, é a pesquisa-ação que tem real mente ajudado a construir o conhecimento.

Inserir o processo na realidade local

Provavelmente, uma das principais diferenças entre as experiências do Cariri e do território do Alto Sertão Piauí/Pernambuco foi a maior inserção desta no territó- 
rio. A integração depende das articulações com outras organizações do território e da legitimidade que estas atribuem à formação. N o TASPP, a ligação com o fórum social era constituinte do projeto inicial, trazendo uma legitimidade forte à formação e dando uma visibilidade aos projetos desenvolvidos pelos alunos. Isso permitiu fortalecer mais o capital institucional.

No C ariri, a formação se fechou sobre si mesmo. A forte dinâmica de grupo desenvolvida dentro da U nicampo isolou os atores do território. Tendo construído novos valores e novas solidariedades, os alunos não se reconheciam mais nas motivações dos outros atores do território. $\mathrm{N}$ ão sabiam como agir para desenvolver projetos conforme os princípios da U nicampo e sentiam-se presos frente os atores políticos. A pesar de uma motivação importante para agir, num primeiro tempo, os atores tiveram dificuldades para se situar em relação às outras organizações e influenciar as políticas territoriais. Esta foi uma grande limitação do processo. E a associação dos alunos que, progressivamente, conseguiu superar em parte o problema. Ainda assim, ela levou vários anos para encontrar uma legitimidade dentro as organizações do território.

I sso mostra a importância de trabalhar dentro dos dispositivos de governança para os outros atores perceberem o interesse da formação: não é suficiente melhorar as competências individuais, é também necessário trabalhar com as organizações do território. É também importante acompanhar os atores formados no longo prazo. No caso da formação no TASPP, os membros da associação dos antigos alunos podiam beneficiar-se de uma formação contínua sobre o processo de governança, o papel do fórum, a gestão de conflitos e a organização de uma associação.

\subsection{ENSINAMENTOS PARA LIGAR MELHOR OS PROCESSOS} DE FORMAÇÃO E DE DESENVOLVIMENTO TERRITORIAL

A análise das experiências permite dar subsídios para uma melhor articulação entre formação e desenvolvimento territorial. $\mathrm{N}$ os dois exemplos, pode se ver a importância do processo pedagógico organizado em etapas. 0 quadro seguinte detal ha estas etapas, mostrando as ligações com o tipo de capital, o processo de aprendizagem e as metodologias utilizadas.

A apresentação aparece esquemática, pois o processo é complexo e cada etapa contribui, de fato, aos diferentes tipos de capitais. D estacamos, pelo menos, o tipo de capital mais específico de cada etapa. N um primeiro momento, antes mesmo do início da formação, numa fase de concepção, é importante entender melhor os processos de desenvolvimento territorial em curso, 0 jogo dos atores, as práticas das diferentes instituições e o funcionamento dos dispositivos de governança mesmo. E sse trabalho tem que permitir entender melhor os problemas e diagnosticar como um dispositivo de construção de conhecimentos pode apoiar as atividades dos dispositivos de governança. Esta fase éfundamental para dar uma legitimidade institucional ao dispositivo de formação. Os alunos e os principais eixos do conteúdo pedagógico têm que ser escolhidos entre os membros do dispositivo de governança e da equipe pedagógica.

$\mathrm{N} o$ desenrolamento das atividades de formação, a primeira fase de conscientização dos participantes corresponde a um trabal ho em torno dos conceitos de desenvolvimento, de campesinato e das alternativas que oferecem um projeto camponês. Faz com que os participantes tenham novas referências e motivações para se organizar. E uma aprendizagem de tipo dupla volta, o que permite a elaboração de valores comuns, de um quadro mínimo de trabalho e de um contrato pedagógico. Ações coletivas de pesquisa têm que ser desenvolvidas para conhecer melhor o território incluído do ponto institucional, para fortalecer a identidade e para ganhar conhecimentos úteis à fase seguinte.

A construção de um projeto comum é a segunda fase. Ela se desenvolve pela confrontação dos conceitos (o projeto camponês) com as realidades institucionais e do campo. E a fase de reflexão sobre as possibilidades de construção de projetos individuais, coletivos e territoriais. 0 s estudantes aprendem a desenvolver o pensamento crítico, a arte da experimentação e da avaliação das experiências que lhes permitam reforçar a sua visão coletiva (dupla volta), mas também e, sobretudo, prática do que pode ser um modelo de desenvolvimento camponês. Pensamos que momentos de debate desses projetos nas instâncias de governança ajudam a adquirir legitimidade, mas permite também ajustes com relação às políticas publicas ou todos outros elementos que poderiam jogar na implementação desses projetos.

$\mathrm{N}$ a fase seguinte, de implementação dos projetos individuais e coletivos mesmos, os alunos aplicam o modelo camponês e mobilizam os conhecimentos e saberes adquiridos. D esenvolvem competências específicas necessárias (técnicas, gestão, relacionamento) para isso. 
Tabela 1. Etapas do processo de construção de um dispositivo de formação

\begin{tabular}{|c|c|c|c|}
\hline Etapas & Que capital? & Que processo? & 0 que fazer? \\
\hline Etapa 1: Constituição de um grupo & $\begin{array}{l}\text { Construir um capital social: linguagem } \\
\text { comum, valores compartilhados }\end{array}$ & Dupla volta, para definir valores comuns & $\begin{array}{l}\text { Atores variados escolhidos e } \\
\text { comprometidos. }\end{array}$ \\
\hline $\begin{array}{l}\text { Etapa 2: Definição de um projeto } \\
\text { territorial comum }\end{array}$ & $\begin{array}{l}\text { A partir do capital social, definir um } \\
\text { projeto adequado à realidade }\end{array}$ & Dupla volta & Problematização, pesquisa-ação \\
\hline Etapa 3: Implementação do projeto & $\begin{array}{l}\text { Capital humano, para implementar os } \\
\text { projetos; Capital institucional: fortalecer } \\
\text { as parcerias para a implementação }\end{array}$ & $\begin{array}{l}\text { Única volta, para reforçar as } \\
\text { competências e saberes-fazeres dos } \\
\text { atores }\end{array}$ & $\begin{array}{l}\text { Treinamentos, experimentações inseridas } \\
\text { na realidade }\end{array}$ \\
\hline Etapa 4: Avaliação & Capital institucional que pode evoluir & $\begin{array}{l}\text { Tripla volta: dar-se um quadro de } \\
\text { aprendizagem para continuar o ciclo de } \\
\text { gestão social }\end{array}$ & $\begin{array}{l}\text { Espaços de reflexão abertos a outros } \\
\text { atores }\end{array}$ \\
\hline
\end{tabular}

$M$ as eles têm que inscrever os projetos no contexto territorial. Muitas vezes, as relações não são simples. Os estudantes podem descobrir que o projeto camponês, como pensado e aplicado, não é compartilhado ou, se é, não é operacionalizado da maneira que eles acham que deve ser. D ai a importância da negociação desses projetos para ganhar ainda mais legitimidade institucional. A criação de uma associação dos alunos é muitas vezes necessária para fortal ecer o capital institucional e atuar como parceiro reconhecido no território. Confrontando com as visões e as práticas dos outros atores, os al unos fortalecem seus próprios valores e as aprendizagens numa pratica técnica, social e institucional.

A avaliação é a fase seguinte. Falar de avaliação não significa um julgamento pontual sobre as escolhas efetuadas, mas é a capacidade de construir, de modo permanente, sentidos a ações, o que supõe sua análise crítica e a compreensão das situações nas quais elas se inserem. Assim, os alunos têm que avaliar as ações a partir da visão de longo prazo e do seu nível de envolvimento no desenvolvimento territorial, incluindo o quadro de aprendizagem mesmo. Esta questão, que valoriza a aprendizagem de tripla volta, levanta a questão da análise do processo da aprendizagem em tempo longo, para avaliar se o treinamento teve um impacto real (efeito trampolim), ou apenas um efeito pontual e anedótico.

$\mathrm{N}$ a replicação de tais processos, 0 caráter inovador deve ser ressaltado. São experiências pedagógicas que dispunham de meios conseqüentes. 0 processo pedagógico permitiu desenvolver aprendizagens de qualidade. Para a sua replicação, um dispositivo de construção de conhecimentos tem que valer-se de um corpo docente com competência de alto nível e isso questiona a maneira de construir tais competências. Pensamos que uma dinâmica de troco entre as experiências antigas e novas, incluindo os al unos, possa ser uma pista de solução interessante.
4. CONCLUSÃO: COMO INTERLIGAR DISPOSITIVOS DE PRODUÇÃO DE COMPETÊNCIAS E DISPOSITIVOS DE GOVERNANÇA?

Q uerendo entender os fatores que determinam a capacidade de uma formação para influenciar as dinâmicas de desenvolvimento territorial, mostramos a importância de construir em conjunto o capital humano, social e institucional. A idéia de capital induz uma idéia de processo, com construção e acumulação (na formação) e mobilização (em projetos). A construção requer um itinerário pedagógico adequado. D epende de como o conhecimento é co-construído durante o processo, mas também da diversidade dos atores envolvidos.

A mobilização depende principalmente do contexto no qual se insere a formação: as instituições presentes, os jogos de atores, as relações de poder. Considerando esse aspecto, é preciso desenvolver antes, durante e após a formação, ligações com as outras organizações do território para dar-Ihe legitimidade, eficiência e capacidade de ação. N este sentido, nos referimos ao conceito de "capabilidade" mencionado na introdução: a construção de competências é necessária, mas é preciso criar as condições para que essas competências possam ser realmente val orizadas e utilizadas.

Assim, para real mente favorecer as dinâmicas territoriais, parece necessário pensar conjuntamente os dispositivos de governança e os dispositivos de produção de competências. 0 desafio de um dispositivo, na acepção de Foucault (1975), é organizar processos que permitem que múltiplos atores chegassem a um acordo sobre objetivos comuns, geran do normas aceitas e legitimadas, traduzindo-se em regras e implementando essas regras (Rey-Valette et al., 2008).

Estes dispositivos podem ser dispositivos de governança, de negociação, mas também de gestão da infor- 
mação ou de educação. M as antes tudo, sempre são dispositivos de diálogo que permitem aprendizagens progressivas dos atores envolvidos. Por conseguinte, é necessário estudar a melhor forma de ligar os diferentes tipos de dispositivos: as aprendizagens realizadas dentro de um dispositivo devem ser aproveitadas para as reflexões dentro do outro. Assim, um problema que surge no âmbito do dispositivo de governança deve ser tratado no dispositivo de produção do conhecimento para desenvolver informações adequadas para resolver este problema. Por outro lado, se os atores envolvidos no dispositivo de produção de conhecimento desenvolvem novas práticas adaptadas ao território, é importante que estas sejam depois debatidas dentro do dispositivo de governança.

Assim, é necessário que uma formação se adapte, baseando-se em experimentações sociais, abordando questões específicas a uma situação determinada, mobilizando técnicas de pesquisa. 0 desafio é multiplicar as experimentações, referenciá-las, avaliá-las e debater os seus resultados nos fóruns de discussão de políticas públicas para que elas contribuam efetivamente para as dinâmicas territoriais.

\section{REFERÊNCIAS BIBLIOGRÁFICAS}

ALBALADEJ0,C.; CASABIAN CA, F. (eds.) Larecherche-action. Ambitions, pratiques, débats, IN RA, Paris. 1997

AM OUREUX, G. L'analyse de la demande dans la relation d'accompagnement. Actualitédela Formation Permanente, vol 178,2002 , p. 20-24.

ARG YRIS, C. ; SCH ÖN , D .A. Organizational learning: A the ory of action perspective, Addison-Wesley, Reading (M assasuchets USA) $1978,344 p$.

ARGYRIS, C. Savoir pour agir. Surmonter les obstacles à I'apprentissageorganisationnel, I nterÉ ditions, Paris. 1995

ARGYRIS, C. SCH ÖN ,D O Organizational learning II: Theory, method and practice, Addison Wesley, Reading (M ass). 1996

BATESO N , G . Stepsto an E cology of M ind: Collected Essaysin Anthropology, Psychiatry, Evolution, and E pistemology, Chandler Press. 1972
BECKER, G.S.. H uman Capital: A Theoretical and Empirical Analysis, with Special Reference to Education, U niversity of Chicago Press, 1993, 390 p.

BREWE, A. A guide to M arx's Capital, Cambridge U niversity Press. 1984

CALL ON , M . (ed.). La ascienceet ses réseaux. Génèseet circulation desfaitsscientifiques, $L$ adécouverte/Conseil del'Europe/ UNESC0.1989

CAN IE LLO,M .; TON N EAU, J.-P. A pedagogia dauniversidadecamponesa. RededeE ducação do Semi-Árido, vol 1(1), 2006 , p. 11-29.

COUDEL, E. Formation et apprentissages pour le dével oppe ment territorial : Regards croisés entreéconomiede la connaissance et sciences degestion. Réflexion à partir d'une U niversité Paysanneau Brésil., http://tel.archives-ouvertes.fr/tel-00371589/ $\mathrm{fr} / \mathrm{T}$ hèsed'économie., M ontpellier SupAgro, 2009, 386p.

DEFFON TAINES,J-P.; M ARCELPOIL,E.; MOQUAY,P..Le développementterritorial: unediversitéd'interprétations. In: LARDON , S. ; M AUREL, P.; PIVETEAU, V. (eds.); Représentations spatial eset dével oppementterritorial. Bilan d'expériencesetperspectivesméthodol ogiques,H ermès, Paris, 2001, p. 39-56.

FOLDY, E.G .; CREED, D. A ction learning, Fragmentation, and the Integration of Single, D ouble, and Triple L oop C hange.T he Journal of Applied Behavioral Science, vol 35(2), 1999, p. 207-227.

FOU CAULT, M. Surveiller et punir: naissance de la prison, Gallimard, Paris, 1975, 360p.

GRAN OVETTER, M . E conomic Action and Social Structure: TheProblem of Embeddedness. American J ournal of Sociology, vol 91(3), 1985, p.481-510.

H ATCHUEL, A. Intervention Research and theProduction of Knowledge. In: Cow up aTree. Knowing and L earning for Change in Agriculture. Case studies from Industrialised Countries, LEARN group (ed.), IN RA éditions, Paris, 2000, p.55-68.

KN OWLES,M. TheAdultL earner: A N eglected Species, Gulf Publishing, H ouston.1990 
KRISH N A, A. Creating and $H$ arnessing Social C apital, In: DASG UPTA, P., SERAG ELDIN , I. (eds.), Social Capital: a Multi-faceted Perspective, World Bank. 1997

LAVERACK,G ; LABONTE, R.A planningframework forcommunity empowerment goals within health promotion. $\mathrm{H}$ ealth Policy Plan 15(3), 2000, p. 255-262.

LARD ON ,S.; M OQUAY. P.; POSS, Y. D éveloppementterritorial etdiagnostic prospectif. Réflexionsautour du viaducdeM iIlau, coll. Essai, La Tour d'Aigues, . 2007, 382 p.

LARDON,S.;TON NEAU, J-P ;RAYMOND,R.;CHIA, E.; CARON , P.. D ispositifs de gouvernance territorialedurable en agriculture. Analyse de trois situations en France et au Brésil. N orois, vol 04(209), 2008, p. 17-36.

LIN , N . Buildinga N etworkT heory of Social Capital. In: LIN , N.; COOK, K.; BU RT, R.S. (eds.) Social Capital. Theory and Research.,AldineTransaction, N ew Brunswick,N ew Jersey, 2005, p. 3-29.

M DA/SDT . ReferênciasparaumaE stratégiadeD esenvolvimento Rural Sustentável no Brasil. Brasília/D F, marçode2005. Série DocumentosSD T: número 1.

PERREN OUD ,P.T heK ey to Social Fields: Competencies of an Autonomous Actor, In: D efining and Selecting Key Competencies. In: RYCHEN , D.S.; SAGALN IK , L.H . (eds.), H ogrefe\& H uber Publishers, Gottingen, 2001, p. 121-149.

PIRAUX, M. ; TON NEAU, J-P ; AZEVEDO S. G. ; SOUZA, C. M. . L edéveloppementterritorial durable: lecas d'uneexpériencedansleN ordestedu Brésil. Colloquedel'ASRD LF deG re noble- Chambéry (11-13juillet 2007). G renoble, France. 2007
PUT N AM , R. The prosperous community. Social capital and publiclife. TheAmerican Prospect, vol 4(13), 1993,P. 35-42.

REY-VALETTE ,H .; LARD ON , S.; CH IA, E. Governance: Institutional and learning plans facilitating the appropriation of sustainable development. International Journal of Sustainable Development, vol 11(2-3-4). 2008

ROM M E, G.; VAN WITTEL OOST UIJN , A. Circular organizing and tripleloop learning.J ournal of $O$ rganizational $C$ hange Management, vol 12(5), 1999, p. 439-454.

SCH U LTZ, T. Investment in H uman Capital. American E conomic Review, vol LI, 1961, p. 1-17.

SDT/M D A. ReferênciasparaumaE stratégiadeD esenvolvimento Rural Sustentável noBrasil,SérieD ocumentosdoSDT: número 1, M inistério do D esenvolvimento Agrario, Brasília. 2005a.

SDT /M DA, ReferênciasparaaG estão Social deTerritóriosRurais, SérieD ocumentosdoSD T: D ocumento I nstitucional $\mathrm{N} \cong 3$, M inistério do D esenvolvimento Agrario, Brasília. . 2005b.

SEN , A. D evelopment as C apability Expansion. Journal of $D e-$ velopment Planning, vol 19, 1989, p. 41-58.

TURCOTTE, M-F. ; ANT ON OVA, S. ; CLEGG, S. Managing learning societally, L es cahiers de la CRSD D, Collection Recherche, Vol. 14-2007, U nivesitédu Q uébec, M ontréal.

Zarifian P. 2001. Lemodèledelacompétence, É ditionsLiaisons, Paris, 2007. 